\title{
A generalization of $\omega$-subdivision ensuring convergence of the simplicial algorithm
}

\author{
Takahito Kuno* \\ Graduate School of Systems and Information Engineering \\ University of Tsukuba, Tsukuba, Ibaraki 305-8573, Japan, \\ and \\ Tomohiro Ishihama \\ NS Solutions Corporation, Chuo, Tokyo 104-8280, Japan.
}

April 1, 2015

Revised: December 4, 2015

\begin{abstract}
In this paper, we refine the proof of convergence by Kuno-Buckland [7] for the simplicial algorithm with $\omega$-subdivision and generalize their $\omega$-bisection rule to establish a class of subdivision rules, called $\omega$-k-section, which bounds the number of subsimplices generated in a single execution of subdivision by a prescribed number $k$. We also report some numerical results of comparing the $\omega$-k-section rule with the usual $\omega$-subdivision rule.
\end{abstract}

Key words: Global optimization, strictly convex maximization, branch-and-bound, simplicial algorithm, $\omega$-subdivision.

\section{Introduction}

The simplicial algorithm is a kind of branch-and-bound algorithm proposed by Horst in 1976 [2] to solve convex maximization problems, and now counted among the most popular algorithms in global optimization $[3,4,12,17]$ along with the conical algorithm developed by Tuy [15]. In the branching process, whereas the latter uses polyhedral cones, the simplicial algorithm uses a set of simplices to partition the feasible set; and in the bounding process, the algorithm computes an upper bound of the objective function by maximizing its concave envelope on each simplex, which is subdivided to refine the partition if the upper bound is large

\footnotetext{
${ }^{*}$ The author was partially supported by a Grant-in-Aid for Scientific Research (C) 25330022 from the Japan Society for the Promotion of Sciences. E-mail: takahito@cs.tsukuba.ac.jp
} 
enough. As a rule for subdivision, Horst used a simple bisection that bisects each simplex across its longest edge, and gave a convergence proof for his algorithm. Although this proof contains a flaw, Thoai-Tuy [14] found and fixed it later, and also introduced the concept of exhaustiveness as a sufficient condition for the convergence. On the other hand, in his conical algorithm, Tuy utilized a byproduct of the bounding process to subdivide each cone without a guarantee of convergence. Even for the simplicial algorithm, we can apply a similar rule, which subdivides each simplex radially outward from the maximum point of the concave envelope obtained as a byproduct in the bounding process. This so-called $\omega$-subdivision rule is not exhaustive, and the convergence of both algorithms according to it remained open until Jaumard-Meyer [5] and Locatelli [9] proved it for the conical algorithm independently in 1998, 1999, and Locatelli-Raber [10, 11] did for the simplicial algorithm in 2000. Preceding their proofs by nearly a decade, Tuy showed in [16] that the conical algorithm with $\omega$-subdivision converges if a certain nondegeneracy condition holds (see also [4, 17]). Kuno-Ishihama [8] showed that a more moderate condition holds always and guarantees the convergence for the conical algorithm. In a similar way, Kuno-Buckland [7] proved the convergence for the simplicial algorithm, but instead allowed an error in the feasibility of the algorithm output within a specified tolerance. They also provided another subdivision rule, called $\omega$-bisection, combining $\omega$-subdivision and bisection, and reported in $[7,8]$ that it improves the computational efficiency of both algorithms.

In this paper, we refine the proof of convergence by Kuno-Buckland in [7] for the simplicial algorithm with $\omega$-subdivision and ensure the feasibility of the algorithm output. Furthermore, we generalize the $\omega$-bisection rule and establish a new class of subdivision rules, called $\omega$ - $k$-section, which bounds the number of subsimplices generated in a single execution of subdivision by a prescribed number $k$. In Section 2, we describe the problem settings, characteristics, and outline the simplicial algorithm according to the $\omega$-subdivision rule. In Section 3 , we introduce a new linear programming relaxation for the subproblem associated with each simplex, and investigate some properties of its optimal solution. Based on the findings, we develop the $\omega$ - $k$-section rule in Section 4 , and give a convergence proof for the simplicial algorithm incorporating it. We report some numerical results of comparing the $\omega$ - $k$-section rule with the usual $\omega$-subdivision rule in Section 5, and make concluding remarks in Section 6.

\section{Convex maximization and the simplicial algorithm}

The problem considered in this paper is as follows

$$
\begin{array}{ll}
\text { maximize } & f(\mathbf{x}) \\
\text { subject to } & \mathbf{A} \leq \mathbf{b},
\end{array}
$$


where $\mathbf{A} \in \mathbb{R}^{m \times n}, \mathbf{b} \in \mathbb{R}^{m}$, and $f$ is a convex function defined on an open convex set $S \subset \mathbb{R}^{n}$. Let

$$
D=\left\{\mathbf{x} \in \mathbb{R}^{n} \mid \mathbf{A x} \leq \mathbf{b}\right\} .
$$

We assume the following throughout the paper:

(A1) There is an $n$-simplex $\Delta^{1}$ with vertices $\mathbf{v}_{1}^{1}, \ldots$, and $\mathbf{v}_{n+1}^{1}$ such that $D \subset \Delta^{1} \subset S$.

(A2) The origin $\mathbf{0} \in \mathbb{R}^{n}$ is an interior point of $D$.

(A3) The value of $f$ is nonnegative on $\Delta^{1}$.

The essence of these assumptions is that the domain $S$ of $f$ is large enough and the feasible set $D$ is a bounded polyhedron with nonempty interior. The remaining conditions are imposed merely for simplicity, and can be satisfied through some elementary transformations.

\section{LIPSCHITZ CONTINUITY OF THE OBJECTIVE FUNCTION}

It is known (see, e.g., Theorem 10.4 in [13]) that a convex function is Lipschitzian relative to any closed bounded subset of its domain, and so is $f$ relative to $\Delta^{1}$. Let us specify the value of a Lipschitz constant for $f$ on $\Delta^{1}$, since we need it to guarantee the convergence of the algorithm.

We see from the assumptions (A1) and (A2) that there exists a sufficiently small positive number $\delta \in(0,1)$ satisfying

$$
\mathbf{0} \in D \subset \Delta^{1} \subset(1+\delta) \Delta^{1} \subset S .
$$

Let

$$
U=\max \left\{f(\mathbf{x}) \mid \mathbf{x} \in(1+\delta) \Delta^{1}\right\},
$$

where $(1+\delta) \Delta^{1}=\left\{(1+\delta) \mathbf{x} \mid \mathbf{x} \in \Delta^{1}\right\}$. Then a Lipschitz constant for $f$ on $\Delta^{1}$ is given by

$$
L=\frac{U}{\delta \operatorname{dist}\left(\mathbf{0}, \partial \Delta^{1}\right)}
$$

where $\operatorname{dist}\left(\mathbf{0}, \partial \Delta^{1}\right)$ denotes the distance from $\mathbf{0}$ to the boundary of $\Delta^{1}$.

Proposition 2.1. It holds that

$$
|f(\mathbf{x})-f(\mathbf{y})| \leq L\|\mathbf{x}-\mathbf{y}\|, \quad \forall \mathbf{x}, \mathbf{y} \in \Delta^{1}
$$

Proof. Let $\alpha$ be a lower bound of $f$ on $\Delta^{1}$. Also let $\rho=\delta \operatorname{dist}\left(\mathbf{0}, \partial \Delta^{1}\right)$, and $\beta$ an upper bound of $f$ on $\Delta^{1}+\rho B=\left\{\mathbf{x}+\rho \mathbf{y} \mid \mathbf{x} \in \Delta^{1}, \mathbf{y} \in B\right\}$, where $B$ is the unit closed ball. The proof of 
Theorem 10.4 in [13] shows that the following holds if $\Delta^{1}+\rho B$ is contained in the domain $S$ of $f$ :

$$
|f(\mathbf{y})-f(\mathbf{x})| \leq \frac{\beta-\alpha}{\rho}\|\mathbf{x}-\mathbf{y}\| .
$$

Since $\delta$ is chosen such that $(1+\delta) \Delta^{1} \subset S$, this proposition follows from (3) for $\alpha=0$ and $\beta=U$ if $\Delta^{1}+\rho B \subset(1+\delta) \Delta^{1}$. To show this, suppose that $\Delta^{1}$ is represented as the intersection of $n+1$ halfspaces $F_{j}, j=1, \ldots, n+1$, and let $\rho_{j}=\delta \operatorname{dist}\left(\mathbf{0}, \partial F_{j}\right)$ for each $j$. Using the property of similar triangles with a vertex at $\mathbf{0}$, we see that $(1+\delta) F_{j}=F_{j}+\rho_{j} B$, and hence

$$
(1+\delta) \Delta^{1}=\bigcap_{j=1}^{n+1}\left(F_{j}+\rho_{j} B\right)
$$

We also have $\Delta^{1}+\rho B \subset F_{j}+\rho B \subset F_{j}+\rho_{j} B$. Therefore, $\Delta^{1}+\rho B$ is a subset of $(1+\delta) \Delta^{1}$.

Note that $L$ is not difficult to determine if $\delta$ is given. In fact, $U$ can be obtained by evaluating $f$ at $n+1$ points $(1+\delta) \mathbf{v}_{j}^{1}, j=1, \ldots, n+1$.

\section{OUTLINE OF THE SIMPLICIAL ALGORITHM}

The simplicial algorithm consists of two distinct processes, branching and bounding. In the branching process, the algorithm subdivides $\Delta^{1}$ into a set of subsimplices $\Delta^{i}, i \in \mathscr{I}$, such that

$$
\Delta^{1}=\bigcup_{i \in \mathscr{I}} \Delta^{i} ; \quad \text { int } \Delta^{p} \cap \operatorname{int} \Delta^{q}=\emptyset \quad \text { if } p, q \in \mathscr{I} \text { and } p \neq q
$$

where $\mathscr{I}$ is an (infinite) index set, and int - denotes the set of interior points. In the bounding process, the algorithm examines whether $\Delta^{i}$ can contain an optimal solution of (1) for each $i \in \mathscr{I}$. This is usually accomplished by using the concave envelope $g^{i}$ of $f$ on $\Delta^{i}$, i.e., the pointwise infimum over all concave overestimators of $f$ on $\Delta^{i}$. In our case where $f$ is convex, $g^{i}$ is an affine function which coincides with $f$ at the vertices of $\Delta^{i}$. Since $D \cap \Delta^{i}$ is a polytope, linear programming finds a maximum point of $g^{i}$ in $D \cap \Delta^{i}$. Let us denote it by $\boldsymbol{\omega}^{i}$. Then we have

$$
g^{i}\left(\boldsymbol{\omega}^{i}\right) \geq g^{i}(\mathbf{x}) \geq f(\mathbf{x}), \quad \forall \mathbf{x} \in D \cap \Delta^{i} .
$$

If $g^{i}\left(\boldsymbol{\omega}^{i}\right) \leq f\left(\mathbf{x}^{*}\right)$ for the best feasible solution $\mathbf{x}^{*}$ of (1) obtained in the process, then $\Delta^{i}$ is removed from further consideration, because it contains no feasible solution better than $\mathbf{x}^{*}$. If not, $\Delta^{i}$ is returned to the branching process and again subdivided for re-examination. Unlike discrete optimization problems, no matter how many times the branching and bounding processes are repeated, $D \cap \Delta^{i}$ can contain infinitely many feasible solutions. In that case, the algorithm generates an infinite sequence of simplices in a nested fashion:

$$
\Delta^{i}=\Delta^{i_{1}} \supset \cdots \supset \Delta^{i_{r}} \supset \Delta^{i_{r+1}} \supset \cdots
$$


where $\Delta^{i_{r+1}}$ is a subsimplex obtained by subdividing $\Delta^{i_{r}}$.

The convergence of the simplicial algorithm depends largely on how to subdivide the simplex $\Delta^{i}$ which is given as $\operatorname{conv}\left\{\mathbf{v}_{j}^{i} \mid j=1, \ldots, n+1\right\}$, the convex hull of $n+1$ vertices. The simplest subdivision rule is bisection, where the longest edge of $\Delta^{i}$ is cut at the midpoint. Then $\Delta^{i}$ itself is divided into two subsimplices of the same volume. According to this rule, the sequence (5) gradually shrinks to a single point, where $f$ agrees with its concave envelope. This exhaustiveness guarantees that the incumbent $\mathbf{x}^{*}$ converges to a globally optimal solution of (1). Another often-used alternative is $\omega$-subdivision. The simplex $\Delta^{i}$ is subdivided radially from $\boldsymbol{\omega}^{i}$ into up to $n+1$ subsimplices. The $\omega$-subdivision rule has been said to be empirically more efficient than bisection. The theoretical convergence, however, was an open question for some decades until Locatelli-Raber proved it in 2000 [10, 11].

In either rule, once a subdivision point $\mathbf{u}^{i} \in \Delta^{i}$ is selected, $\Delta^{i}$ is subdivided radially outward from $\mathbf{u}^{i}$ into

$$
\Delta_{j}^{i}=\operatorname{conv}\left\{\mathbf{v}_{1}^{i}, \ldots, \mathbf{v}_{j-1}^{i}, \mathbf{u}^{i}, \mathbf{v}_{j+1}^{i}, \ldots, \mathbf{v}_{n+1}^{i}\right\}, \quad j \in J^{i},
$$

where $J^{i}$ is an index set such that $j \in J^{i}$ if $\mathbf{u}^{i}$ is affinely independent of $\mathbf{v}_{1}^{i}, \ldots, \mathbf{v}_{j-1}^{i}, \mathbf{v}_{j+1}^{i}, \ldots$, $\mathbf{v}_{n+1}^{i}$. In the case of bisection, $\mathbf{u}^{i}$ is the midpoint of the longest edge, and obviously $\mathbf{u}^{i}=\boldsymbol{\omega}^{i}$ under the $\omega$-subdivision rule. We therefore say that $\Delta^{i}$ is subdivided via $\mathbf{u}^{i}$ into (6). In the next section, we will discuss how to select this subdivision point $\mathbf{u}^{i}$ in order to make the difference between $f$ and $g^{i}$ at $\mathbf{u}^{i}$ converge to zero.

\section{Linear programming relaxation}

Let us suppose that the simplicial algorithm is infinite and generates a sequence of nested simplices:

$$
\Delta^{1} \supset \cdots \supset \Delta^{i} \supset \Delta^{i+1} \supset \cdots
$$

where $\Delta^{i+1}$ is an $n$-simplex obtained by subdividing $\Delta^{i}$ via a point $\mathbf{u}^{i} \in \Delta^{i}$. Associated with each $\Delta^{i}$ is a subproblem

$$
\left(\mathrm{P}_{\Delta^{i}}\right) \mid \begin{array}{ll}
\text { maximize } & f(\mathbf{x}) \\
\text { subject to } & \mathbf{x} \in D \cap \Delta^{i} .
\end{array}
$$

Replacing $f$ with its concave envelope $g^{i}$ on $\Delta^{i}$, we have the usual linear programming relaxation

$$
\begin{array}{ll}
\text { maximize } & g^{i}(\mathbf{x}) \\
\text { subject to } & \mathbf{x} \in D \cap \Delta^{i},
\end{array}
$$

the optimal value of which is an upper bound for $\left(\mathrm{P}_{\Delta^{i}}\right)$. 


\section{SOLUTION PROPERTIES}

For the vertices $\mathbf{v}_{j}^{i}, j=1, \ldots, n+1$, of $\Delta^{i}$, let

$$
\mathbf{d}^{i}=\left[f\left(\mathbf{v}_{1}^{i}\right), \ldots, f\left(\mathbf{v}_{n+1}^{i}\right)\right], \quad \mathbf{V}^{i}=\left[\mathbf{v}_{1}^{i}, \ldots, \mathbf{v}_{n+1}^{i}\right]
$$

The concave envelope $g^{i}$ is then given explicitly as

$$
g^{i}(\mathbf{x})=\mathbf{c x}+c_{0}
$$

in terms of the solution $\left[\mathbf{c}, c_{0}\right]$ to a linear system:

$$
\left[\mathbf{c}, c_{0}\right]\left[\begin{array}{c}
\mathbf{V}^{i} \\
\mathbf{e}
\end{array}\right]=\mathbf{d}^{i}
$$

where $\mathbf{e} \in \mathbb{R}^{n+1}$ is the all-ones row vector. Without knowing $\left[\mathbf{c}, c_{0}\right]$, however, we can solve (8) by solving an equivalent linear programming problem with variables $\lambda \in \mathbb{R}^{n+1}$ :

$$
\mid \begin{aligned}
& \operatorname{maximize} \mathbf{d}^{i} \boldsymbol{\lambda} \\
& \text { subject to } \quad \mathbf{A V}^{i} \boldsymbol{\lambda} \leq \mathbf{b}, \quad \mathbf{e} \boldsymbol{\lambda}=1, \quad \boldsymbol{\lambda} \geq \mathbf{0} .
\end{aligned}
$$

In this paper, introducing an additional variable $\tau$, we modify (9) and solve instead

$$
(\mathrm{PL}) \mid \begin{aligned}
& \operatorname{maximize} \quad \mathbf{d}^{i} \boldsymbol{\lambda}-M \tau \\
& \text { subject to } \quad \mathbf{A} \mathbf{V}^{i} \boldsymbol{\lambda} \leq \mathbf{b}, \quad \mathbf{e} \boldsymbol{\lambda}+\tau=1, \quad \boldsymbol{\lambda} \geq \mathbf{0}, \quad \tau \geq 0,
\end{aligned}
$$

where $M$ is a constant determined by the Lipschitz constant $L$ of $f$ given in (2) and the initial simplex $\Delta^{1}=\operatorname{conv}\left\{\mathbf{v}_{j}^{1} \mid j=1, \ldots, n+1\right\}$, to satisfy

$$
M \geq L \max \left\{\left\|\mathbf{v}_{j}^{1}\right\| \mid j=1, \ldots, n+1\right\}
$$

Since $(\boldsymbol{\lambda}, \tau)=(\mathbf{0}, 1)$ is a feasible solution and the objective function is bounded from above by

$$
U^{i}=\max \left\{f\left(\mathbf{v}_{j}^{i}\right) \mid j=1, \ldots, n+1\right\},
$$

problem (PL) always has an optimal solution, which we denote by $\left(\boldsymbol{\lambda}^{i}, \tau^{i}\right)$.

Lemma 3.1. The optimal value of $(P L)$ is an upper bound for $\left(P_{\Delta^{i}}\right)$, i.e.,

$$
\mathbf{d}^{i} \lambda^{i}-M \tau^{i} \geq f(\mathbf{x}), \quad \forall \mathbf{x} \in D \cap \Delta^{i}
$$

Moreover, $D \cap \Delta^{i}=\emptyset$ if $\tau^{i}>\delta$.

Proof. Assume that $D \cap \Delta^{i} \neq \emptyset$, and choose any point $\mathbf{x}$ in it. Then (9) is feasible and has an 
optimal solution $\boldsymbol{\lambda}^{\prime}$, which satisfies $\mathbf{d}^{i} \boldsymbol{\lambda}^{\prime} \geq f(\mathbf{x})$. This implies the first claim because $\left(\boldsymbol{\lambda}^{\prime}, 0\right)$ is a feasible solution and $\left(\boldsymbol{\lambda}^{i}, \tau^{i}\right)$ is an optimal solution of (PL). We also have $\mathbf{d}^{i} \boldsymbol{\lambda}^{i}-M \tau^{i} \geq 0$ by assumption (A3), and hence

$$
\tau^{i} \leq \frac{\mathbf{d}^{i} \boldsymbol{\lambda}^{i}}{M} \leq \frac{U^{i} \delta \operatorname{dist}\left(\mathbf{0}, \partial \Delta^{1}\right)}{U \max \left\{\left\|\mathbf{v}_{j}^{1}\right\| \mid j=1, \ldots, n+1\right\}} \leq \delta,
$$

by noting $\operatorname{dist}\left(\mathbf{0}, \partial \Delta^{1}\right) \leq \max \left\{\left\|\mathbf{v}_{j}^{1}\right\| \mid j=1, \ldots, n+1\right\}$ because $\mathbf{0} \in \Delta^{1}$.

Let $\boldsymbol{\omega}^{i}=\mathbf{V}^{i} \boldsymbol{\lambda}^{i}$. If $\tau^{i}=0$, then $\boldsymbol{\omega}^{i}$ is an optimal solution of (8) and a feasible solution of $\left(\mathrm{P}_{\Delta^{i}}\right)$. If $\tau^{i}>0$, then $\boldsymbol{\omega}^{i}$ is a feasible solution of the original problem (1). Although $\boldsymbol{\omega}^{i}$ might not be a point in $D \cap \Delta^{i}$, we can assume

$$
\mathbf{d}^{i} \lambda^{i}-M \tau^{i} \geq f\left(\boldsymbol{\omega}^{i}\right)
$$

because otherwise $\Delta^{i}$ contains no feasible solutions better than $\omega^{i}$ and can be removed from consideration. Let

$$
\Delta_{+}^{i}=\operatorname{conv}\left\{\mathbf{v}_{j}^{i} \mid j \in J^{i}\right\}, \quad J^{i}=\left\{j \mid \lambda_{j}^{i}>0\right\},
$$

both of which we can assume to be nonempty; otherwise, $D \cap \Delta^{i}=\emptyset$ by Lemma 3.1, and $\Delta^{i}$ can again be removed. By linear programming duality, there is an optimal solution to the dual of (PL),

$$
\text { (DL) } \mid \begin{array}{ll}
\text { minimize } & \boldsymbol{\mu} \mathbf{b}+v \\
\text { subject to } & \boldsymbol{\mu} \mathbf{A} \mathbf{V}^{i}+v \mathbf{e} \geq \mathbf{d}^{i}, \quad v \geq-M, \quad \boldsymbol{\mu} \geq \mathbf{0} .
\end{array}
$$

Let $\left(\boldsymbol{\mu}^{i}, v^{i}\right)$ be an optimal solution of (DL).

Lemma 3.2. It holds that

$$
\boldsymbol{\mu}^{i} \mathbf{A x}+v^{i} \geq g^{i}(\mathbf{x}), \quad \forall \mathbf{x} \in \Delta^{i}
$$

In particular,

$$
\boldsymbol{\mu}^{i} \mathbf{A} \mathbf{x}+v^{i}=g^{i}(\mathbf{x}) \quad \text { if } \mathbf{x} \in \Delta_{+}^{i} .
$$

Proof. Let $\mathbf{x}$ be any point in $\Delta^{i}$. There exists some $\boldsymbol{\lambda} \geq \mathbf{0}$ such that $\mathbf{e} \boldsymbol{\lambda}=1$ and $\mathbf{x}=\mathbf{V}^{i} \boldsymbol{\lambda}$. Since $g^{i}$ is an affine function, we have

$$
g^{i}(\mathbf{x})=\mathbf{d}^{i} \boldsymbol{\lambda} \leq\left(\boldsymbol{\mu}^{i} \mathbf{A} \mathbf{V}^{i}+v^{i} \mathbf{e}\right) \boldsymbol{\lambda}=\boldsymbol{\mu}^{i} \mathbf{A} \mathbf{x}+\boldsymbol{v}^{i},
$$

by noting the first set of constraints of (DL). If $\mathbf{x} \in \Delta_{+}^{i}$, then $\lambda_{j}=0$ for each $j \notin J^{i}$, and besides

$$
d_{j}^{i}=\boldsymbol{\mu}^{i} \mathbf{A} \mathbf{v}_{j}^{i}+v^{i}, \quad \forall j \in J^{i},
$$

by complementary slackness between $\left(\boldsymbol{\lambda}^{i}, \tau^{i}\right)$ and $\left(\boldsymbol{\mu}^{i}, \boldsymbol{v}^{i}\right)$. These, together with (11), imply the latter assertion. 
Lemma 3.3. If $p \leq i$, then

$$
\boldsymbol{\mu}^{p} \mathbf{A} \mathbf{x}+v^{p} \geq \boldsymbol{\mu}^{i} \mathbf{A} \mathbf{x}+v^{i}, \quad \forall \mathbf{x} \in \Delta_{+}^{i}
$$

Proof. We see from the preceding lemma that the right-hand side of the inequality coincides with the concave envelope $g^{i}$ of $f$ on $\Delta_{+}^{i}$. In contrast, the left-hand side is just an overestimator of $f$ on $\Delta_{+}^{i} \subset \Delta^{p}$.

Lemma 3.4. There exists a constant $C$ such that

$$
\left\|\boldsymbol{\mu}^{i} \mathbf{A}\right\| \leq C, \quad i=1,2, \ldots
$$

Proof. If $\left\|\boldsymbol{\mu}^{i} \mathbf{A}\right\|=0$ for every $i$, nothing to prove. Choose any $i$ such that $\left\|\boldsymbol{\mu}^{i} \mathbf{A}\right\|>0$. Let

$$
G=\left\{\mathbf{x} \in \mathbb{R}^{n} \mid \boldsymbol{\mu}^{i} \mathbf{A} \mathbf{x} \leq \boldsymbol{\mu}^{i} \mathbf{b}\right\}
$$

The distance from $\mathbf{0}$ to the boundary of this half space is $\operatorname{dist}(\mathbf{0}, \partial G)=\boldsymbol{\mu}^{i} \mathbf{b} /\left\|\boldsymbol{\mu}^{i} \mathbf{A}\right\|$. Since $\mathbf{0}$ is an interior point of $D$, which is a subset of $G$, the distance between $\mathbf{0}$ and $\partial D$ is at most $\operatorname{dist}(\mathbf{0}, \partial G)$. Therefore,

$$
\left\|\boldsymbol{\mu}^{i} \mathbf{A}\right\|=\frac{\boldsymbol{\mu}^{i} \mathbf{b}}{\operatorname{dist}(\mathbf{0}, \partial G)} \leq \frac{\boldsymbol{\mu}^{i} \mathbf{b}}{\operatorname{dist}(\mathbf{0}, \partial D)} \leq \frac{U^{i}-v^{i}}{\operatorname{dist}(\mathbf{0}, \partial D)} \leq \frac{U+M}{\operatorname{dist}(\mathbf{0}, \partial D)},
$$

where the last inequality follows from the constraint $v \geq-M$ of (DL). Let $C$ denote the last term in this chain of inequalities. Then $C$ is a constant for each instance of (1) and satisfies the inequality in the lemma.

\section{BEHAVIOR OF NESTED SIMPLICES}

In the rest of this section, we assume in the sequence (7) that the subdivision point $\mathbf{u}^{i}$ of $\Delta^{i}$ is selected in the face $\Delta_{+}^{i}$ for each $i$. According to this subdivision rule, $\mathbf{v}_{j}^{i}$ remains in $\Delta^{i+1}$ as a vertex unless $\mathbf{v}_{j}^{i}$ belongs to $\Delta_{+}^{i}$. We also have the following convergence result:

Theorem 3.5. If $\Delta^{i+1}$ is obtained by subdividing $\Delta^{i}$ via a point $\mathbf{u}^{i} \in \Delta_{+}^{i}$ for $i=1,2, \ldots$, then

$$
\liminf _{i \rightarrow \infty}\left(g^{i}\left(\mathbf{u}^{i}\right)-f\left(\mathbf{u}^{i}\right)\right)=0
$$

To prove this theorem, we need one more lemma:

Lemma 3.6. If $\Delta^{i+1}$ is obtained by subdividing $\Delta^{i}$ via a point $\mathbf{u}^{i} \in \Delta_{+}^{i}$ for $i=1,2, \ldots$, then there exists a subsequence $\left\{i_{r} \mid r=1,2, \ldots\right\}$ such that

$$
\mathbf{u}^{i_{r}} \in \Delta_{+}^{i_{r}} \cap \Delta_{+}^{i_{r+1}}, \quad r=1,2, \ldots
$$


Proof. We first show that, for all but finitely many $i$,

$$
\exists p(i)>i, \quad \mathbf{u}^{i} \in \Delta_{+}^{p(i)}
$$

If not, there exists an infinite subsequence $\left\{i_{s} \mid s=1,2, \ldots\right\}$ such that (12) fails for $i=i_{s}$. Since $\mathbf{u}^{i_{s}}$ is inherited as a vertex from $\Delta^{i_{s}+1}$ to all its descendants, the $i_{n+1}$ th simplex $\Delta^{i_{n+1}}$ is spanned only by $\mathbf{u}^{i_{1}}, \ldots \mathbf{u}^{i_{n+1}}$, at least one of which must be a point in $\Delta_{+}^{i_{n+1}}$. This is a contradiction, and hence there exists an integer $q$ such that (12) holds for every $i>q$. Choose an index $i>q$ as $i_{1}$, and denote by $i_{2}$ the index $p\left(i_{1}\right)$ corresponding to $i_{1}$. Then denote $p\left(i_{2}\right)$ by $i_{3}$. Repeating this process yields an infinite subsequence $\left\{i_{r} \mid r=1,2, \ldots\right\}$, which satisfies the condition.

We are now ready to prove Theorem 3.5.

Proof of Theorem 3.5. Consider the same subsequence $\left\{i_{r} \mid r=1,2, \ldots\right\}$ as in Lemma 3.6. Let

$$
H^{r}=\left\{(\mathbf{x}, y) \in \mathbb{R}^{n} \times \mathbb{R} \mid \boldsymbol{\mu}^{i_{r}} \mathbf{A x}+v^{i_{r}} \geq y\right\}
$$

and let

$$
\mathbf{z}^{r}=\left(\mathbf{u}^{i_{r}}, \boldsymbol{\mu}^{i_{r}} \mathbf{A} \mathbf{u}^{i_{r}}+v^{i_{r}}\right) .
$$

While $\mathbf{z}^{r}$ is not a point in $H^{r+1}$, we see from Lemma 3.3 that

$$
\mathbf{z}^{r} \in \bigcap_{s=1}^{r} H^{s}, \quad r=1,2, \ldots
$$

As a consequence of the bounded convergence principle (see e.g., Lemma III.2 in [4]), the distance between $\mathbf{z}^{r}$ and $H^{r+1}$ converges to zero, and thereby

$$
\operatorname{dist}\left(\mathbf{z}^{r}, \partial H^{r+1}\right) \rightarrow 0, \quad \text { as } r \rightarrow \infty
$$

Since $\partial H^{r+1}=\left\{(\mathbf{x}, y) \in \mathbb{R}^{n} \times \mathbb{R} \mid \boldsymbol{\mu}^{i_{r+1}} \mathbf{A} \mathbf{x}+\boldsymbol{v}^{i_{r+1}}=y\right\}$, we have

$$
\operatorname{dist}\left(\mathbf{z}^{r}, \partial H^{r+1}\right)=\frac{\boldsymbol{\mu}^{i_{r}} \mathbf{A} \mathbf{u}^{i_{r}}+\boldsymbol{v}^{i_{r}}-\boldsymbol{\mu}^{i_{r+1}} \mathbf{A} \mathbf{u}^{i_{r}}-v^{i_{r+1}}}{\left(\left\|\boldsymbol{\mu}^{i_{r+1}} \mathbf{A}\right\|^{2}+1\right)^{1 / 2}} .
$$

Also, it follows from Lemmas 3.2 and 3.6 that

$$
\boldsymbol{\mu}^{i_{r}} \mathbf{A} \mathbf{u}^{i_{r}}+v^{i_{r}}=g^{i_{r}}\left(\mathbf{u}^{i_{r}}\right), \quad \boldsymbol{\mu}^{i_{r+1}} \mathbf{A} \mathbf{u}^{i_{r}}+v^{i_{r+1}}=g^{i_{r+1}}\left(\mathbf{u}^{i_{r}}\right) .
$$

Furthermore, $\mathbf{u}^{i_{r}}$ is a vertex of $\Delta^{i_{r+1}}$, and hence $g^{i_{r+1}}\left(\mathbf{u}^{i_{r}}\right)=f\left(\mathbf{u}^{i_{r}}\right)$. These, together with (14), imply

$$
\operatorname{dist}\left(\mathbf{z}^{r}, \partial H^{r+1}\right)=\frac{g^{i_{r}}\left(\mathbf{u}^{i_{r}}\right)-f\left(\mathbf{u}^{i_{r}}\right)}{\left(\left\|\boldsymbol{\mu}^{i_{r+1}} \mathbf{A}\right\|^{2}+1\right)^{1 / 2}}
$$


From (13), (15) and Lemma 3.4, we have the following for some constant $C$ :

$$
g^{i_{r}}\left(\mathbf{u}^{i_{r}}\right)-f\left(\mathbf{u}^{i_{r}}\right) \leq\left(C^{2}+1\right)^{1 / 2} \operatorname{dist}\left(\mathbf{z}^{r}, \partial H^{r+1}\right) \rightarrow 0, \quad \text { as } r \rightarrow \infty,
$$

Thus, we complete the proof because $g^{i}\left(\mathbf{u}^{i}\right)-f\left(\mathbf{u}^{i}\right) \geq 0$ for every $i$, and its lower limit cannot be below zero.

Theorem 3.5 alone is not sufficient to ensure the convergence of the algorithm to an optimal solution of the target problem (1). To accomplish this, we need to further restrict the selection of $\mathbf{u}^{i}$ for each $i$. In the next section, we extend the idea used for $\omega$-bisection, a hybrid of $\omega$ subdivision and bisection proposed in [7], and develop a new class of subdivision rule called $\omega$ - $k$-section, which bounds the number of subsimplices generated in a single execution of subdivision by a prescribed integer $k \in\{2, \ldots, n+1\}$.

\section{Simplicial algorithm based on $\omega$ - $k$-section}

In establishing the $\omega$ - $k$-section rule, we need to make an additional assumption:

(A4) The objective function $f$ of (1) is strictly convex.

Namely, if $\mathbf{x}, \mathbf{y} \in S$ and $\mathbf{x} \neq \mathbf{y}$, we assume

$$
f[(1-\lambda) \mathbf{x}+\lambda \mathbf{y}]<(1-\lambda) f(\mathbf{x})+\lambda f(\mathbf{y}), \quad \forall \lambda \in(0,1)
$$

This implies that $f$ does not agree with its concave envelope $g^{i}$ on $\Delta^{i}$ except at the vertices $\mathbf{v}_{j}^{i}$, $j=1, \ldots, n+1$.

For a prescribed integer $k \in\{2, \ldots, n+1\}$, let

$$
k^{i}=\min \left\{k,\left|J^{i}\right|\right\}, \quad i=1,2, \ldots
$$

In the $\omega$ - $k$-section rule, the subdivision point $\mathbf{u}^{i}$ of $\Delta^{i}$ is selected in a $\left(k^{i}-1\right)$-face of $\Delta_{+}^{i}$. Therefore, we first need to determine a subset $K^{i} \subset J^{i}$ with $\left|K^{i}\right|=k^{i}$ such that $\mathbf{u}^{i} \in \operatorname{conv}\left\{\mathbf{v}_{j}^{i} \mid\right.$ $\left.j \in K^{i}\right\}$. Before describing it, let us see how the sequence (7) of nested simplices behaves under the assumption (A4) if such a subdivision rule is applied.

Lemma 4.1. Let $\Delta^{i+1}$ be obtained by subdividing $\Delta^{i}$ via a point $\mathbf{u}^{i} \in \operatorname{conv}\left\{\mathbf{v}_{j}^{i} \mid j \in K^{i}\right\}$ for $i=1,2, \ldots$ Then there exists a subsequence $\left\{i_{r} \mid r=1,2, \ldots\right\}$ such that $K^{i_{r}}=K^{0}$ for every $r$, and

$$
\mathbf{v}_{j}^{i_{r}} \rightarrow \mathbf{v}_{j}^{0}, \quad j \in K^{0}, \quad \mathbf{u}^{i_{r}} \rightarrow \mathbf{u}^{0} \in\left\{\mathbf{v}_{j}^{0} \mid j \in K^{0}\right\}, \quad \text { as } r \rightarrow \infty .
$$

Proof. Since $k^{i}$ can take only $k$ integral values, there exists a positive integer $p \leq k$ such that $k^{i}=p$ for infinitely many $i$. The number of $(p-1)$-faces of $\Delta^{i}$ is also finite. Passing to a 
suitable subsequence $\left\{i_{r} \mid r=1,2, \ldots\right\}$ and renumbering, we have $K^{i_{r}}=K^{0}=\{1, \ldots, p\}$ for every $r$, and besides

$$
\mathbf{v}_{j}^{i_{r}} \rightarrow \mathbf{v}_{j}^{0} \in \Delta^{1}, \quad j=1, \ldots, p, \quad \mathbf{u}^{i_{r}} \rightarrow \mathbf{u}^{0} \in \operatorname{conv}\left\{\mathbf{v}_{j}^{0} \mid j=1, \ldots, p\right\}
$$

because $\mathbf{u}^{i}$ and $\mathbf{v}_{j}^{i}$ are generated in the compact set $\Delta^{1}$. If the value of $\min \left\{\left\|\mathbf{v}_{j}^{i_{r}}-\mathbf{u}^{i_{r}}\right\| \mid j=\right.$ $1, \ldots, p\}$ converges to zero, we have $\mathbf{u}^{0} \in\left\{\mathbf{v}_{j}^{0} \mid j=1, \ldots, p\right\}$, by taking a further subsequence if necessary. Suppose on the contrary that there exists a number $\sigma>0$ such that for any $r$,

$$
\exists s>r, \quad \min \left\{\left\|\mathbf{v}_{j}^{i_{s}}-\mathbf{u}^{i_{s}}\right\| \mid j=1, \ldots, p\right\} \geq \sigma
$$

Note that the subdivision discussed here is a special case of that in Theorem 3.5, and we can assume that

$$
g^{i_{r}}\left(\mathbf{u}^{i_{r}}\right)-f\left(\mathbf{u}^{i_{r}}\right) \rightarrow 0, \quad \text { as } r \rightarrow \infty .
$$

Consider the following problem with variables $\mathbf{y}_{j} \in \mathbb{R}^{n}, j=1, \ldots, p$, and $\zeta \in \mathbb{R}^{p}$ :

$$
\begin{array}{|ll}
\operatorname{minimize} & \sum_{j=1}^{p} \zeta_{j} f\left(\mathbf{y}_{j}\right)-f\left(\sum_{j=1}^{p} \zeta_{j} \mathbf{y}_{j}\right) \\
\text { subject to } & \left\|\mathbf{y}_{j}-\sum_{\ell=1}^{p} \zeta_{\ell} \mathbf{y}_{\ell}\right\| \geq \sigma, \quad \mathbf{y}_{j} \in \Delta^{1}, \quad j=1, \ldots, p \\
& \mathbf{e} \boldsymbol{\zeta}=1, \quad \zeta \geq \mathbf{0} .
\end{array}
$$

Under assumption (A4), the value of the objective function vanishes if and only if $\zeta_{j}=1$ for some $j$. However, such a solution is excluded from the feasible set by the constraint $\left\|\mathbf{y}_{j}-\sum_{\ell=1}^{p} \zeta_{\ell} \mathbf{y}_{\ell}\right\| \geq \sigma$. As a consequence, if (18) is feasible, it has an optimal solution with positive optimal value, say $\eta>0$. Since $\mathbf{u}^{i_{s}} \in \operatorname{conv}\left\{\mathbf{v}_{j}^{i_{s}} \mid j \in K^{0}\right\}$, there exists some $\boldsymbol{\zeta}^{\prime} \geq \mathbf{0}$ such that $\mathbf{e} \boldsymbol{\zeta}^{\prime}=1$ and $\mathbf{u}^{i_{s}}=\sum_{j=1}^{p} \zeta_{j}^{\prime} \mathbf{v}_{j}^{i_{s}}$. It follows from (16) that $\left(\mathbf{v}_{1}^{i_{s}}, \ldots, \mathbf{v}_{p}^{i_{s}}, \boldsymbol{\zeta}^{\prime}\right)$ is a feasible solution of (18), and hence

$$
g^{i_{s}}\left(\mathbf{u}^{i_{s}}\right)-f\left(\mathbf{u}^{i_{s}}\right)=\sum_{j=1}^{p} \zeta_{j}^{\prime} f\left(\mathbf{v}_{j}^{i_{s}}\right)-f\left(\sum_{j=1}^{p} \zeta_{j}^{\prime} \mathbf{v}_{j}^{i_{s}}\right) \geq \eta>0 .
$$

This is a contradiction to (17). Thus, (16) is false, and $\mathbf{u}^{0} \in\left\{\mathbf{v}_{j}^{0} \mid j=1, \ldots, p\right\}$. 


\section{ESTABLISHMENT OF THE $\omega$ - $k$-SECTION RULE}

Let us establish the $\omega$ - $k$-section rule based on the observation in Lemma 4.1. For each subset $K \subset J^{i}$ with $|K|=k^{i}$, let

$$
\mathbf{u}_{K}^{i}=\sum_{j \in K} \bar{\lambda}_{j}^{i} \mathbf{v}_{j}^{i}, \quad \bar{\lambda}_{j}^{i}=\lambda_{j}^{i} / \sum_{\ell \in K} \lambda_{\ell}^{i}, \quad j \in K
$$

Then $\bar{\lambda}_{j}^{i}>0$ for each $j \in K$ and $\sum_{j \in K} \bar{\lambda}_{j}^{i}=1$; and therefore $\mathbf{u}_{K}^{i}$ lies in the relative interior of the $\left(k^{i}-1\right)$-face $\operatorname{conv}\left\{\mathbf{v}_{j}^{i} \mid j \in K\right\}$ of $\Delta_{+}^{i}$. Also let

$$
\rho_{K}=\min \left\{\left\|\mathbf{v}_{j}^{i}-\mathbf{u}_{K}^{i}\right\| \mid j \in K\right\}
$$

Among $\mathbf{u}_{K}^{i}$ 's, we select as the subdivision point $\mathbf{u}^{i}$ the one with the largest $\rho_{K}$, i.e., indexed by

$$
K^{i} \in \arg \max \left\{\rho_{K}\left|K \subset J^{i},\right| K \mid=k^{i}\right\} .
$$

Then $\Delta^{i}$ is subdivided via $\mathbf{u}^{i}=\mathbf{u}_{K^{i}}^{i}$ in the $\left(k^{i}-1\right)$-face $\operatorname{conv}\left\{\mathbf{v}_{j}^{i} \mid j \in K^{i}\right\}$ into

$$
\Delta_{j}^{i}=\operatorname{conv}\left\{\mathbf{v}_{1}^{i}, \ldots, \mathbf{v}_{j-1}^{i}, \mathbf{u}^{i}, \mathbf{v}_{j+1}^{i}, \ldots, \mathbf{v}_{n+1}^{i}\right\}, \quad j \in K^{i}
$$

some one of which is adopted as the successor $\Delta^{i+1}$ of $\Delta^{i}$.

If the sequence (7) of nested simplices is generated under this $\omega$-k-section rule, we have the following results.

Lemma 4.2. Let $\Delta^{i+1}$ be obtained by subdividing $\Delta^{i}$ according to (19)-(22) for $i=1,2, \ldots$ There exists a subsequence $\left\{i_{r} \mid r=1,2, \ldots\right\}$ such that $J^{i_{r}}=J^{0}$ for every $r$, and

$$
\boldsymbol{\lambda}^{i_{r}} \rightarrow \lambda^{0}, \quad \tau^{i_{r}} \rightarrow \tau^{0}, \quad \text { as } r \rightarrow \infty
$$

for some $\lambda^{0}$ and $\tau^{0}$ satisfying

$$
\sum_{j \in J^{0}} \lambda_{j}^{0}+\tau^{0}=1, \quad \lambda^{0} \geq \mathbf{0}, \quad 0 \leq \tau^{0} \leq \delta
$$

Moreover, for each $K \subset J^{0}$ with $|K|=\min \left\{k,\left|J^{0}\right|\right\}$,

$$
\mathbf{v}_{j}^{i_{r}} \rightarrow \mathbf{v}_{j}^{0}, \quad j \in K, \quad \mathbf{u}_{K}^{i_{r}} \rightarrow \mathbf{u}_{K}^{0} \in\left\{\mathbf{v}_{j}^{0} \mid j \in K\right\}, \quad \text { as } r \rightarrow \infty .
$$

In particular, for each $j \in K$, if $\lambda_{j}^{0}>0$, then $\mathbf{v}_{j}^{0}=\mathbf{u}_{K}^{0}$.

Proof. Let $\left\{i_{r} \mid r=1,2, \ldots\right\}$ be a subsequence as in Lemma 4.1. Then $K^{i_{r}}$ is the same set $K^{0}$ with $\left|K^{0}\right|=p \leq k$ for every $r$. The superset $J^{i_{r}}$ of $K^{i_{r}}$ is also drawn from the finite set, 
and hence we can assume $J^{i_{r}}=J^{0}$ for every $r$. Since $\lambda_{j}^{i_{r}}>0$ if and only if $j \in J^{0}$, we have $\sum_{j \in J^{0}} \lambda_{j}^{i_{r}}+\tau^{i_{r}}=1$; and besides $\tau^{i_{r}} \leq \delta$ by Lemma 3.1. These observations lead to the first assertion.

For each $K \subset J^{0}$ with $|K|=p$, we have

$$
\mathbf{v}_{j}^{i_{r}} \rightarrow \mathbf{v}_{j}^{0} \in \Delta^{1}, \quad j \in K, \quad \mathbf{u}_{K}^{i_{r}} \rightarrow \mathbf{u}_{K}^{0} \in \operatorname{conv}\left\{\mathbf{v}_{j}^{0} \mid j \in K\right\}
$$

While (20) and (21) imply $\min \left\{\left\|\mathbf{v}_{j}^{i_{r}}-\mathbf{u}_{K}^{i_{r}}\right\| \mid j \in K\right\} \leq \rho_{K^{i_{r}}}$, we see from Lemma 4.1 that $\rho_{K^{i_{r}}} \rightarrow 0$. Therefore, $\mathbf{u}_{K}^{0}$ lies in $\left\{\mathbf{v}_{j}^{0} \mid j \in K\right\}$. To prove the rest, suppose $\sum_{j \in K} \lambda_{j}^{0}>0$ and $\mathbf{u}_{K}^{0}=\mathbf{v}_{q}^{0}$ for some $q \in K$. Since $g^{i_{r}}\left(\mathbf{v}_{q}^{i_{r}}\right)=f\left(\mathbf{v}_{q}^{i_{r}}\right)$ for each $r$, we have

$$
\left|g^{i_{r}}\left(\mathbf{u}_{K}^{i_{r}}\right)-f\left(\mathbf{u}_{K}^{i_{r}}\right)\right| \leq\left|g^{i_{r}}\left(\mathbf{u}_{K}^{i_{r}}\right)-g^{i_{r}}\left(\mathbf{v}_{q}^{i_{r}}\right)\right|+\left|f\left(\mathbf{v}_{q}^{i_{r}}\right)-f\left(\mathbf{u}_{K}^{i_{r}}\right)\right| \rightarrow 0 .
$$

However, by the continuity of $f$, we have

$$
\begin{gathered}
g^{i_{r}}\left(\mathbf{u}_{K}^{i_{r}}\right)=\sum_{j \in K} \bar{\lambda}_{j}^{i_{r}} f\left(\mathbf{v}_{j}^{i_{r}}\right) \rightarrow \sum_{j \in K} \bar{\lambda}_{j}^{0} f\left(\mathbf{v}_{j}^{0}\right) \\
f\left(\mathbf{u}_{K}^{i_{r}}\right)=f\left(\sum_{j \in K} \bar{\lambda}_{j}^{i_{r}} \mathbf{v}_{j}^{i_{r}}\right) \rightarrow f\left(\sum_{j \in K} \bar{\lambda}_{j}^{0} \mathbf{v}_{j}^{0}\right),
\end{gathered}
$$

where $\bar{\lambda}_{j}^{0}=\lambda_{j}^{0} / \sum_{\ell \in K} \lambda_{\ell}^{0}$. Therefore, if $\lambda_{j}^{0}>0$, it is necessary for (23) that $\mathbf{v}_{j}^{0}=\mathbf{u}_{K}^{0}$ under assumption of (A4).

Lemma 4.3. Let $\Delta^{i+1}$ be obtained by subdividing $\Delta^{i}$ according to (19)-(22) for $i=1,2, \ldots$ Then

$$
\liminf _{i \rightarrow \infty}\left(\mathbf{d}^{i} \lambda^{i}-M \tau^{i}-f\left(\boldsymbol{\omega}^{i}\right)\right)=0
$$

Proof. Let $\left\{i_{r} \mid r=1,2, \ldots\right\}$ be the same sequence used in the previous lemmas. Then we have $J^{i_{r}}=J^{0}$ for each $r$. After renumbering, we can assume that $J^{0}=\{1, \ldots, q\}$ for some integer $q \in[p, n+1]$, where $p=k^{i_{r}}$ for every $r$. First, we will show that for $\ell=p+1, \ldots, q$, if

$$
\mathbf{w}_{\ell-1}^{i_{r}}=\sum_{j=1}^{\ell-1} \lambda_{j}^{i_{r}} \mathbf{v}_{j}^{i_{r}} \rightarrow\left(\sum_{j=1}^{\ell-1} \lambda_{j}^{0}\right) \mathbf{v}_{s}^{0}
$$

for some $s \in\{1, \ldots, \ell-1\}$, then

$$
\mathbf{w}_{\ell}^{i_{r}}=\sum_{j=1}^{\ell} \lambda_{j}^{i_{r}} \mathbf{v}_{j}^{i_{r}} \rightarrow\left(\sum_{j=1}^{\ell} \lambda_{j}^{0}\right) \mathbf{v}_{t}^{0}
$$

for some $t \in\{\ell, s\}$. Note that (24) follows immediately from Lemma 4.2 when $\ell=p$. 
Suppose $\lambda_{\ell}^{0}>0$, because (24) is obvious when $\lambda_{\ell}^{0}=0$. If $\lambda_{j}^{0}=0$ for $j=1, \ldots, \ell-1$, then

$$
\left\|\mathbf{w}_{\ell}^{i_{r}}-\left(\sum_{j=1}^{\ell} \lambda_{j}^{i_{r}}\right) \mathbf{v}_{\ell}^{0}\right\|=\left\|\sum_{j=1}^{\ell-1} \lambda_{j}^{i_{r}}\left(\mathbf{v}_{j}^{i_{r}}-\mathbf{v}_{\ell}^{i_{r}}\right)\right\| \rightarrow 0,
$$

and we have $\mathbf{w}_{\ell}^{i_{r}} \rightarrow\left(\sum_{j=1}^{\ell} \lambda_{j}^{0}\right) \mathbf{v}_{\ell}^{0}$. Even if $\lambda_{j}^{0}>0$ for some $j$, we see from Lemma 4.2 that $\mathbf{v}_{j}^{0}=\mathbf{v}_{\ell}^{0}=\mathbf{u}_{K}^{0}$ for some $K \subset J^{0}$ such that $j, \ell \in K$ and $|K|=p$, and again (25) holds. By induction, there exists an index $t \in J^{0}$ such that

$$
\boldsymbol{\omega}^{i_{r}}=\mathbf{w}_{q}^{i_{r}}=\sum_{j \in J^{0}} \lambda_{j}^{i_{r}} \mathbf{v}_{j}^{i_{r}} \rightarrow \boldsymbol{\omega}^{0}=\left(\sum_{j \in J^{0}} \lambda_{j}^{0}\right) \mathbf{v}_{t}^{0}=\left(1-\tau^{0}\right) \mathbf{v}_{t}^{0} .
$$

For each $r$, let

$$
\overline{\boldsymbol{\omega}}^{i_{r}}=\frac{1}{1-\tau^{i_{r}}} \boldsymbol{\omega}^{i_{r}}
$$

Since $\boldsymbol{\omega}^{i_{r}}$ and $\overline{\boldsymbol{\omega}}^{i_{r}}$ both belong to $\Delta^{1}=\operatorname{conv}\left\{\mathbf{v}_{j}^{1} \mid j=1, \ldots, n+1\right\}$, we see from Proposition 2.1 that

$$
\begin{aligned}
\left|f\left(\overline{\boldsymbol{\omega}}^{i_{r}}\right)-f\left(\boldsymbol{\omega}^{i_{r}}\right)\right| & \leq L\left\|\overline{\boldsymbol{\omega}}^{i_{r}}-\boldsymbol{\omega}^{i_{r}}\right\|=L \tau^{i_{r}}\left\|\overline{\boldsymbol{\omega}}^{i_{r}}\right\| \\
& \leq L \tau^{i_{r}} \max \left\{\left\|\mathbf{v}_{j}^{1}\right\| \mid j=1, \ldots, n+1\right\} \leq M \tau^{i_{r}}
\end{aligned}
$$

and hence

$$
f\left(\boldsymbol{\omega}^{i_{r}}\right) \geq f\left(\overline{\boldsymbol{\omega}}^{i_{r}}\right)-M \tau^{i_{r}}
$$

Taking limits of both sides yields

$$
f\left(\boldsymbol{\omega}^{0}\right) \geq f\left(\mathbf{v}_{t}^{0}\right)-M \tau^{0}
$$

We also have

$$
\mathbf{d}^{i_{r}} \lambda^{i_{r}}=\sum_{j \in J^{0}} f\left(\mathbf{v}_{j}^{i_{r}}\right) \lambda_{j}^{i_{r}} \rightarrow \mathbf{d}^{0} \lambda^{0}=\sum_{j \in J^{0}} f\left(\mathbf{v}_{j}^{0}\right) \lambda_{j}^{0}=f\left(\mathbf{v}_{t}^{0}\right) \sum_{j \in J^{0}} \lambda_{j}^{0}
$$

by noting $\mathbf{v}_{j}^{0}=\mathbf{v}_{t}^{0}$ if $\lambda_{j}^{0}>0$. This, together with (26), implies that

$$
f\left(\boldsymbol{\omega}^{0}\right) \geq \mathbf{d}^{0} \boldsymbol{\lambda}^{0}-M \tau^{0}
$$

because $1-\delta \leq \sum_{j \in J^{0}} \lambda_{j}^{0} \leq 1$ and $f\left(\mathbf{v}_{t}^{0}\right) \geq 0$. In addition to this, the inequality (10) is assumed for every $i$, and hence the assertion follows. 


\section{AlgORITHM AND ITS CONVERGENCE}

Let us incorporate the $\omega$ - $k$-section rule into the simplicial algorithm for solving (1). For a given tolerance $\varepsilon \geq 0$, the algorithm is described as follows.

algorithm simplicial_omega $(D, f, \varepsilon, k)$

determine a simplex $\Delta^{1}=\operatorname{conv}\left\{\mathbf{v}_{j}^{1} \mid j=1, \ldots, n+1\right\} \supset D$ and a constant $\delta \in(0,1)$ such that $D \subset \Delta^{1} \subset(1+\delta) \Delta^{1} \subset S$ for the domain $S$ of $f$;

determine a constant $M \geq L \max \left\{\left\|\mathbf{v}_{j}^{1}\right\| \mid j=1, \ldots, n+1\right\}$ for the Lipschitz constant $L$ of $f$ on $\Delta^{1}$;

$\mathscr{P} \leftarrow \emptyset ; \mathscr{T} \leftarrow\left\{\Delta^{1}\right\} ; \mathbf{x}^{1} \leftarrow \mathbf{0} ; \alpha^{1} \leftarrow f(\mathbf{0}) ; i \leftarrow 1 ;$ stop $\leftarrow$ false $;$

while stop $=$ false do

for each $\Delta=\operatorname{conv}\left\{\mathbf{v}_{j} \mid j=1, \ldots, n+1\right\} \in \mathscr{T}$ do

let $\mathbf{d} \leftarrow\left[f\left(\mathbf{v}_{1}\right), \ldots, f\left(\mathbf{v}_{n+1}\right)\right], \mathbf{V} \leftarrow\left[\mathbf{v}_{1}, \ldots, \mathbf{v}_{n+1}\right]$, and define the relaxed problem (PL)

of the subproblem $\left(\mathrm{P}_{\Delta}\right)$;

solve (PL) and obtain an optimal solution $\left(\boldsymbol{\lambda}_{\Delta}, \tau_{\Delta}\right)$;

if $\tau_{\Delta} \leq \delta$ then

$\boldsymbol{\omega}_{\Delta} \leftarrow \mathbf{V} \boldsymbol{\lambda}_{\Delta} ; \beta_{\Delta} \leftarrow \mathbf{d} \boldsymbol{\lambda}_{\Delta}-M \tau_{\Delta} ;$

if $f\left(\boldsymbol{\omega}_{\Delta}\right)>\alpha^{i}$ then

$\mathbf{x}^{i} \leftarrow \boldsymbol{\omega}_{\Delta} ; \alpha^{i} \leftarrow f\left(\mathbf{x}^{i}\right)$

end if

else

$\mathscr{T} \leftarrow \mathscr{T} \backslash\{\Delta\}$

end if

end for

$\mathscr{P} \leftarrow\left\{\Delta \in \mathscr{P} \cup \mathscr{T} \mid \beta_{\Delta}-\alpha^{i} \geq \varepsilon\right\} ;$

if $\mathscr{P}=\emptyset$ then

stop $\leftarrow$ true;

else

select $\Delta$ with the largest $\beta_{\Delta}$ from $\mathscr{P}$, and let $\mathscr{P} \leftarrow \mathscr{P} \backslash\{\Delta\}$;

$\Delta^{i} \leftarrow \Delta ; \beta^{i} \leftarrow \beta_{\Delta} ; \lambda^{i} \leftarrow \lambda_{\Delta} ; \tau^{i} \leftarrow \tau_{\Delta} ; \boldsymbol{\omega}^{i} \leftarrow \omega_{\Delta} ;$

$J^{i} \leftarrow\left\{j \mid \lambda_{j}^{i}>0\right\} ; k^{i} \leftarrow \min \left\{k,\left|J^{i}\right|\right\}$

determine $K^{i} \subset J^{i}$ with $\left|K^{i}\right|=k^{i}$ and $\mathbf{u}^{i} \in \operatorname{conv}\left\{\mathbf{v}_{j}^{i} \mid j \in K^{i}\right\}$ according to (19)- (21);

subdivide $\Delta^{i}$ via $\mathbf{u}^{i}$ into $k^{i}$ subsimplices $\Delta_{j}^{i}, j \in K^{i}$;

$\mathscr{T} \leftarrow\left\{\Delta_{j}^{i} \mid j \in K^{i}\right\} ; \mathbf{x}^{i+1} \leftarrow \mathbf{x}^{i} ; \alpha^{i+1} \leftarrow \alpha^{i} ; i \leftarrow i+1 ;$

end if

end while

$\mathbf{x}^{*} \leftarrow \mathbf{x}^{i}$;

end. 
Theorem 4.4. Suppose $\varepsilon=0$. If the algorithm simplicial_omega terminates after finitely many iterations, then $\mathbf{x}^{*}$ is an optimal solution of (1). Even if not, every accumulation point of the sequence $\left\{\mathbf{x}^{i} \mid i=1,2, \ldots\right\}$ is an optimal solution.

Proof. If the algorithm terminates, the claim is obvious. Consider the case where simplicial_omega does not terminate. Then it generates at least one infinite sequence of nested simplices. Selecting any one and renumbering, we denote it as $\left\{\Delta^{i} \mid i=1,2, \ldots\right\}$. Since $\alpha^{i}$ is nondecreasing in $i$ and bounded from above by $U^{1}$, it converges to some $\alpha^{0} \in\left[0, U^{1}\right]$. Passing to a suitable subsequence $\left\{i_{r} \mid r=1,2, \ldots\right\}$, we also have $\beta^{i_{r}} \rightarrow \beta^{0}, \boldsymbol{\omega}^{i_{r}} \rightarrow \boldsymbol{\omega}^{0}$, as $r \rightarrow \infty$, and $\beta^{0}=f\left(\boldsymbol{\omega}^{0}\right)$ by Lemma 4.3. However, $f\left(\boldsymbol{\omega}^{i}\right) \leq \alpha^{i} \leq \beta^{i}$ for every $i$, and hence $f\left(\boldsymbol{\omega}^{i_{r}}\right)$ and $\beta^{i_{r}}$ both converge to $\alpha^{0}$.

In order to show

$$
\alpha^{0} \geq f(\mathbf{x}), \quad \forall \mathbf{x} \in D,
$$

suppose $f(\mathbf{y})>\alpha^{0}$ for some $\mathbf{y} \in D$. At iteration $i_{r}$ for each $r$, this feasible solution $\mathbf{y}$ belongs to some simplex $\Delta \in \mathscr{P}$, and we have

$$
\alpha^{0}<f(\mathbf{y}) \leq \beta_{\Delta} \leq \beta^{i_{r}}
$$

by noting that $\Delta^{i_{r}}$ has been selected from $\mathscr{P}$. However, $\beta^{i_{r}} \rightarrow \alpha^{0}$, and hence $f(\mathbf{y})=\alpha^{0}$. This is a contradiction and (27) holds. On the other hand, since $\left\{\mathbf{x}^{i} \mid i=1,2, \ldots\right\}$ is generated in $D \subset \Delta^{1}$, any accumulation point, say $\mathbf{x}^{0}$, belongs to $D$. For any subsequence $\left\{i_{s} \mid s=1,2, \ldots\right\}$ such that $\mathbf{x}^{i_{s}} \rightarrow \mathbf{x}^{0}$, we have

$$
f\left(\mathbf{x}^{i_{s}}\right)=\alpha^{i_{s}} \rightarrow \alpha^{0}, \quad \text { as } s \rightarrow \infty
$$

because $\left\{\alpha^{i} \mid i=1,2, \ldots\right\}$ is a convergent sequence. This, together with (27), implies that $\mathbf{x}^{0}$ is an optimal solution of (1).

Corollary 4.5. If $\varepsilon>0$, the algorithm simplicial_omega terminates with an $\varepsilon$-optimal solution $\mathbf{x}^{*}$ of (1) after finitely many iterations.

Proof. As seen in the proof of Theorem 4.4, if $\varepsilon=0$ and simplicial_omega does not terminate, then

$$
\liminf _{i \rightarrow \infty}\left(\beta^{i}-\alpha^{i}\right)=0 .
$$

For each $\Delta \in \mathscr{P}$, we have $\alpha^{i} \leq \beta_{\Delta} \leq \beta^{i}$. Therefore, $\beta_{\Delta}-\alpha^{i}<\varepsilon$ holds at some iteration $i$ and the algorithm terminates if $\varepsilon>0$. Since $\alpha^{i}=f\left(\mathbf{x}^{i}\right)$, this termination criterion implies the $\varepsilon$-optimality of $\mathbf{x}^{*}=\mathbf{x}^{i}$ for (1). 


\section{Numerical results}

In this section, we report the numerical results of comparing $\omega$-bisection, $\omega$-trisection and $\omega$ quadsection, i.e., $\omega$ - $k$-section for $k=2,3,4$, respectively, with the usual $\omega$-subdivision. The test problem solved using the algorithm simplicial_omega is a convex quadratic maximization problem of the form

$$
\mid \begin{array}{ll}
\operatorname{maximize} & f(\mathbf{x})+\theta \mathbf{c}_{y} \mathbf{y} \\
\text { subject to } & \mathbf{A x}+\mathbf{B y} \leq \mathbf{b}, \quad[\mathbf{x}, \mathbf{y}] \geq \mathbf{0}
\end{array}
$$

where

$$
f(\mathbf{x})=\frac{1}{2} \mathbf{x}^{\top} \mathbf{Q} \mathbf{x}+\mathbf{c}_{x} \mathbf{x} .
$$

To make the feasible set bounded, the vector $\mathbf{b} \in \mathbb{R}^{m}$ was fixed to $[1, \ldots, 1, n]^{\top}$ and all components of the last row of $\mathbf{A} \in \mathbb{R}^{m \times q}$ and $\mathbf{B} \in \mathbb{R}^{m \times(n-q)}$ were set to ones. Other entries of $[\mathbf{A}, \mathbf{B}]$, together with components of $\left[\mathbf{c}_{x}, \mathbf{c}_{y}\right] \in \mathbb{R}^{q} \times \mathbb{R}^{n-q}$, were generated randomly in the interval $[-0.5,1.0]$, so that the percentages of zeros and negative numbers were about $20 \%$ and $10 \%$, respectively. The matrix $\mathbf{Q} \in \mathbb{R}^{q \times q}$ was symmetric, tridiagonal, and the tridiagonal entries were random numbers in $[0.0,1.0]$.

Note that the objective function of (28) can be linearized by replacing only the nonlinear part $f$ with its concave envelope. Therefore, we may implement the branching process in the $\mathbf{x}$-space of dimension $q \leq n$, instead of in the whole space of dimension $n$. Based on this decomposition principle [4], we programmed simplicial_omega in GNU Octave [1], a numerical computing environment similar to MATLAB, and tested it on one core of Intel Core i7 (3.70GHz). In preprocessing, we computed the Lipschitz constant $L$ of $f$ according to (2) with $\delta=10^{-10}$, and used it to determine the constant $M$ needed for defining the relaxed problem $(\mathrm{PL})$. As the procedure for solving (PL), we used the revised simplex algorithm, which was not an optimization toolbox procedure but coded from scratch in Octave. Furthermore, we replaced the bounding criterion $\beta_{\Delta}-\alpha^{i} \geq \varepsilon$ in simplicial_omega with

$$
\beta_{\Delta}-(1+\varepsilon) \alpha^{i} \geq 0
$$

where $\varepsilon$ was set to $10^{-5}$, in order to prevent the convergence from being affected by the magnitude of the optimal value. Let us denote by $2-\mathrm{sec}, 3-\mathrm{sec}, 4-\mathrm{sec}$ and subdiv the program codes of simplicial_omega incorporating $\omega$-bisection, $\omega$-trisection, $\omega$-quadsection and $\omega$-subdivision, respectively. As varying $m, n, q$ and $\theta$, we solved ten instances of (28) and measured the average performance of each code for each set of the parameters.

Figures 1 and 2 plot the changes in the average number of iterations and the average CPU time in seconds, respectively, taken by each program code when the dimensionality $q$ of $\mathbf{x}$ increased from 30 to 60 , with $(m, n, \theta)$ fixed at $(60,100,5.0)$. Figures 3 and 4 show the results when the weight $\theta$ in the objective function changed between 3.0 and 10.0, with 


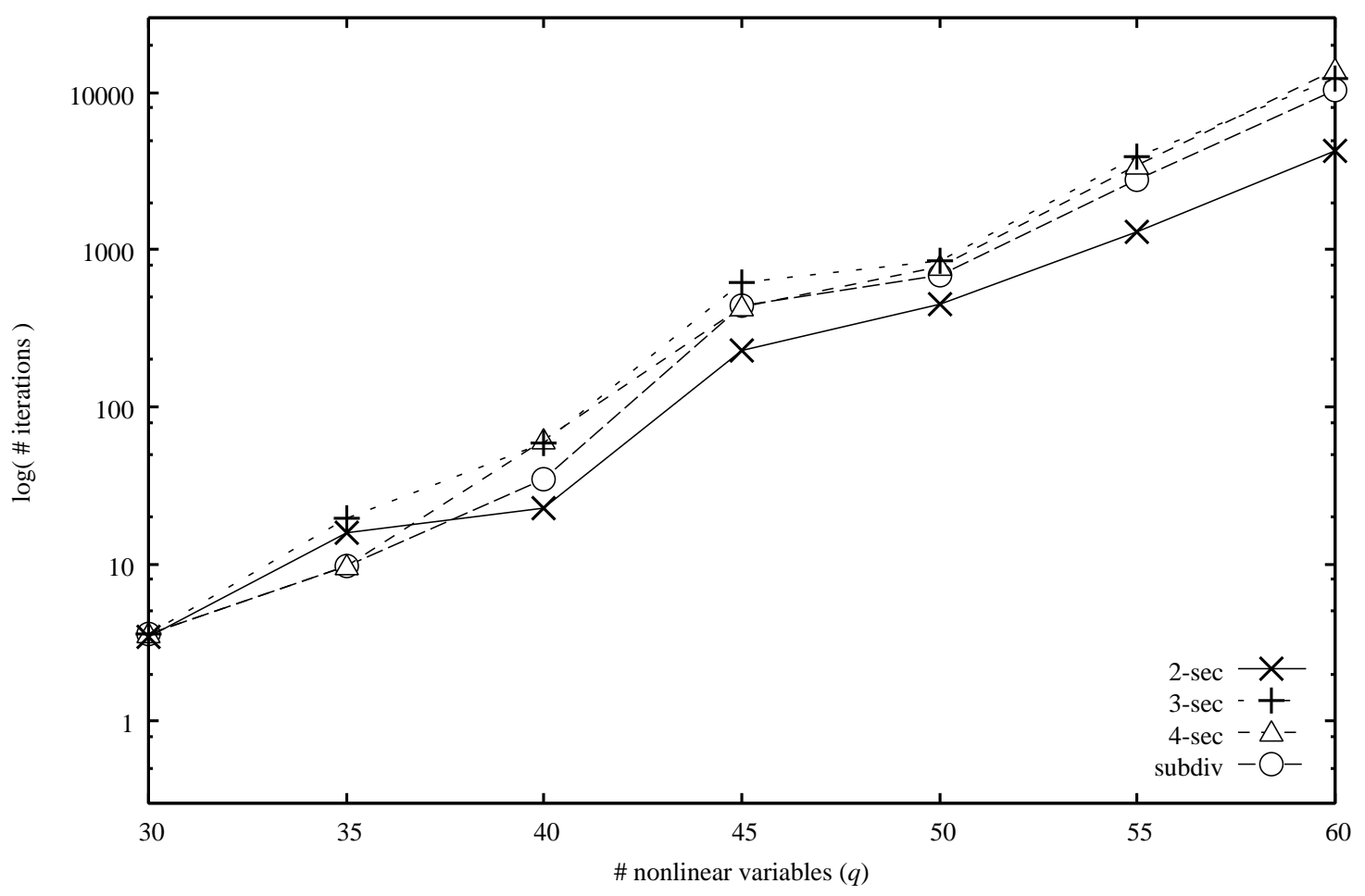

Figure 1: Number of iterations when $(m, n, \theta)=(60,100,5.0)$.

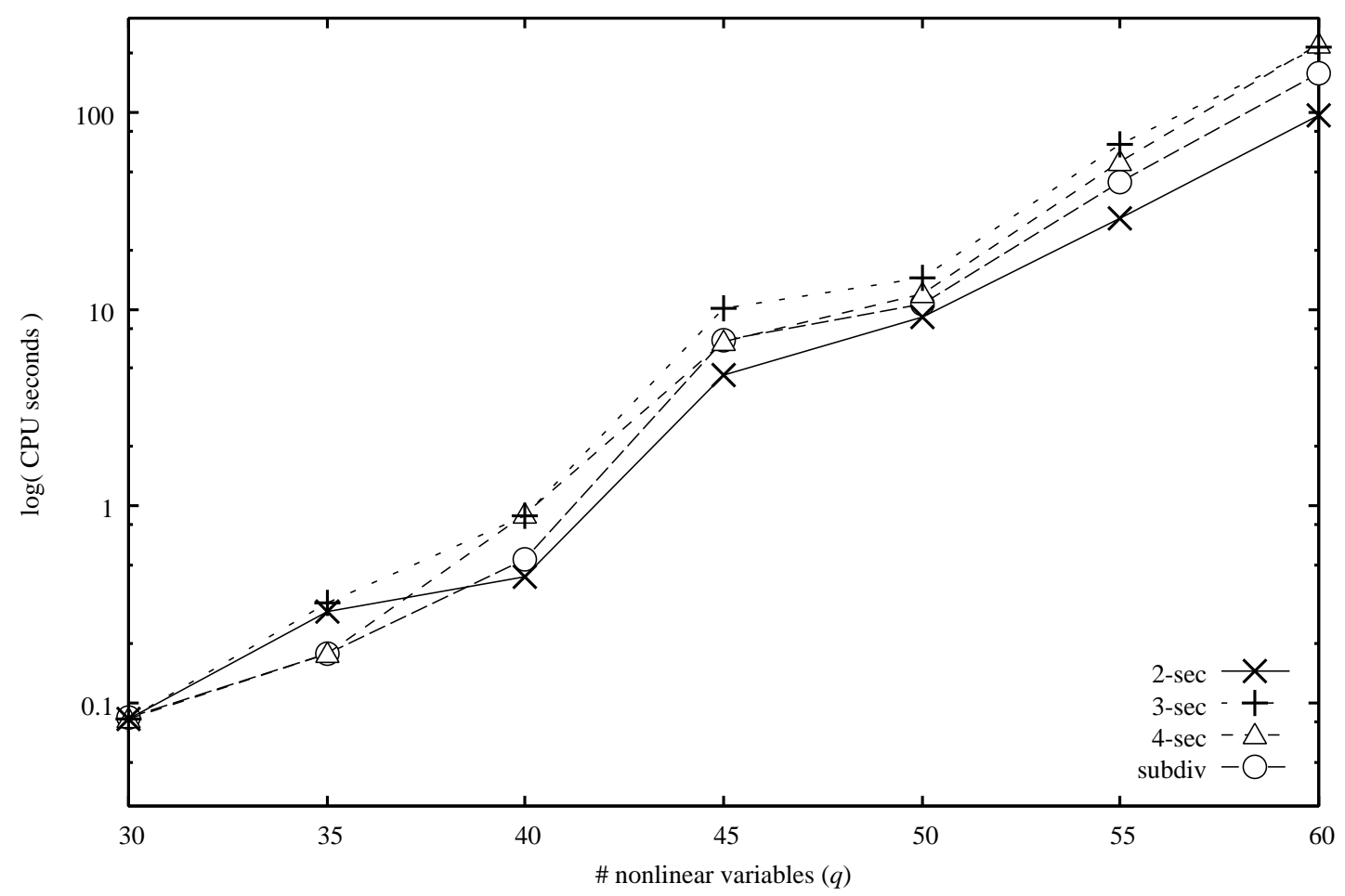

Figure 2: CPU time in seconds when $(m, n, \theta)=(60,100,5.0)$. 


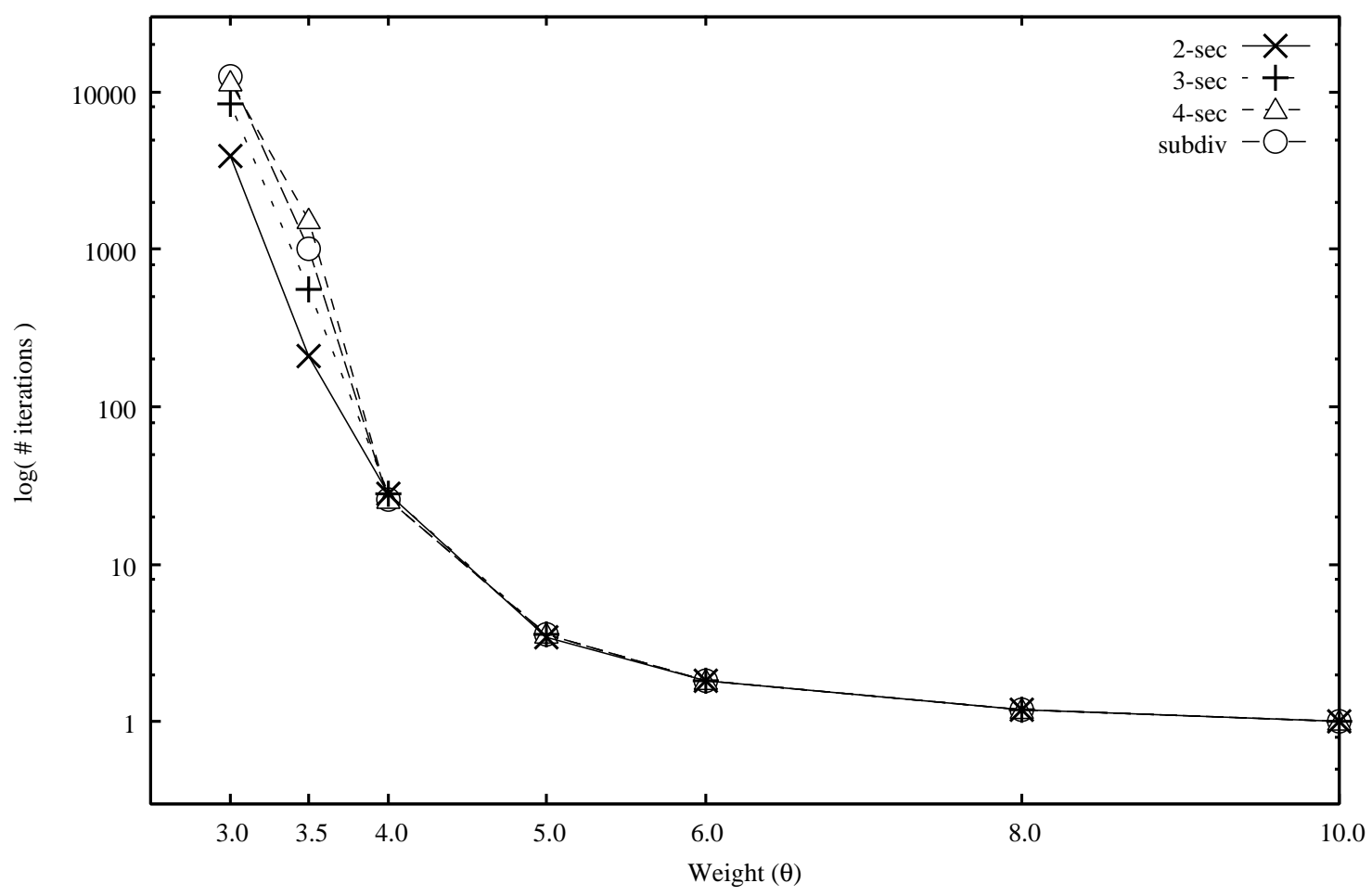

Figure 3: Number of iterations when $(m, n, q)=(60,100,30)$.

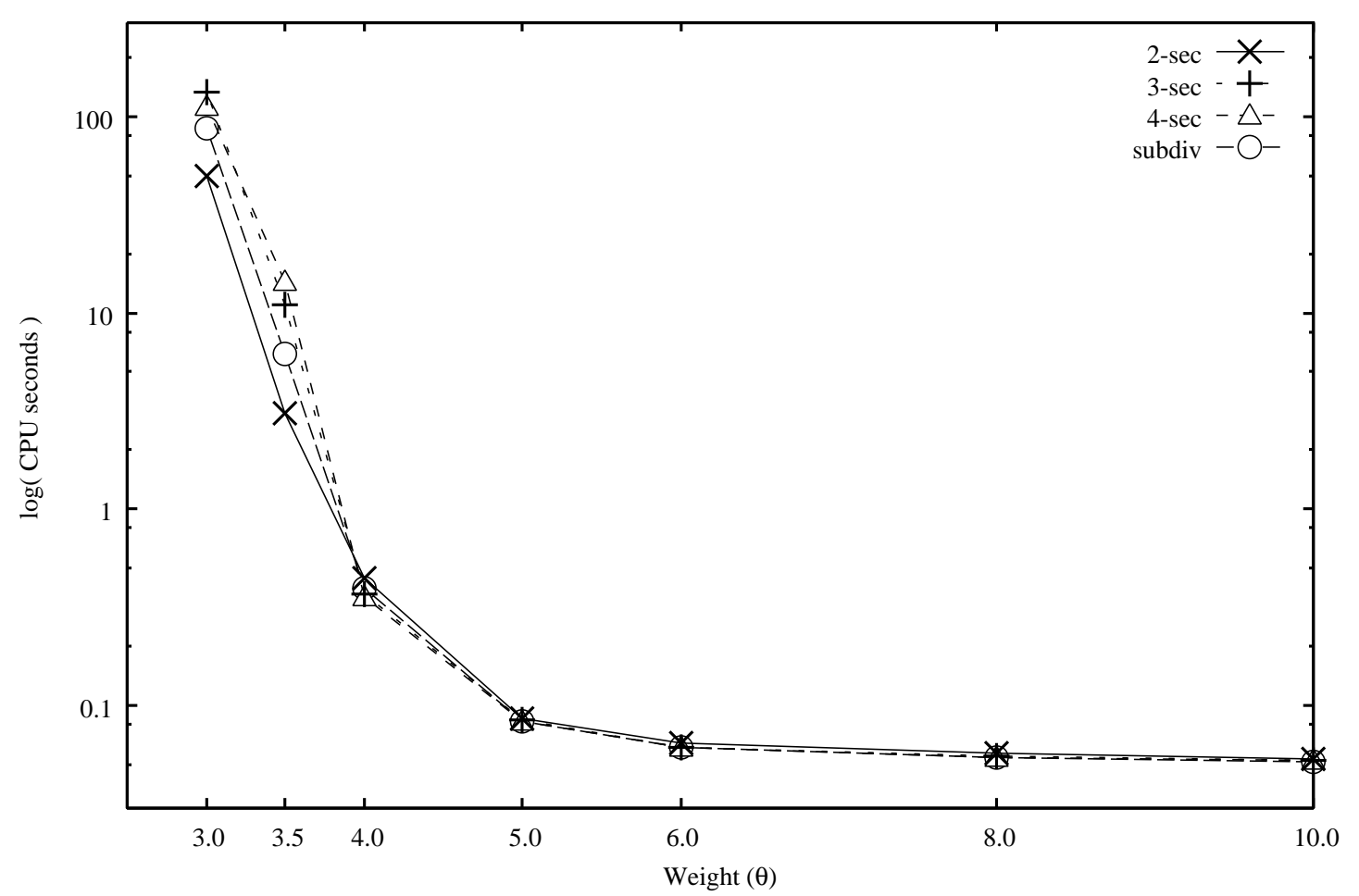

Figure 4: CPU time in seconds when $(m, n, q)=(60,100,30)$. 
Table 1: Computational results of simplicial_omega when $\theta=5.0$.

\begin{tabular}{|c|c|c|c|c|c|c|c|}
\hline \multirow[b]{2}{*}{$m \times n$} & & \multicolumn{2}{|c|}{$q=0.3 n$} & \multicolumn{2}{|c|}{$q=0.4 n$} & \multicolumn{2}{|c|}{$q=0.5 n$} \\
\hline & & $\#$ & time & \# & time & \# & time \\
\hline \multirow[t]{3}{*}{$60 \times 150$} & $2-\sec$ & 5.6 & 0.201 & 13.6 & 0.511 & 74.8 & 2.967 \\
\hline & 3-sec & 3.8 & 0.150 & 30.5 & 0.895 & 236.2 & 7.397 \\
\hline & subdiv & 3.8 & 0.149 & 55.2 & 1.554 & 109.5 & 3.515 \\
\hline \multirow[t]{3}{*}{$90 \times 150$} & $2-\sec$ & 3.0 & 0.234 & 89.6 & 5.207 & 47.8 & 3.465 \\
\hline & $3-\sec$ & 2.8 & 0.220 & 88.1 & 4.289 & 78.1 & 4.490 \\
\hline & subdiv & 2.8 & 0.228 & 89.1 & 4.489 & 125.6 & 6.746 \\
\hline \multirow[t]{3}{*}{$90 \times 200$} & $2-\sec$ & 4.6 & 0.429 & 21.4 & 1.917 & 29.2 & 3.137 \\
\hline & $3-\sec$ & 5.5 & 0.435 & 19.8 & 1.600 & 75.8 & 6.570 \\
\hline & subdiv & 7.9 & 0.540 & 23.2 & 1.760 & 194.5 & 15.55 \\
\hline \multirow[t]{3}{*}{$120 \times 200$} & $2-\mathrm{sec}$ & 2.6 & 0.488 & 8.4 & 1.339 & 13.6 & 2.458 \\
\hline & $3-\sec$ & 2.5 & 0.493 & 8.9 & 1.237 & 17.1 & 2.616 \\
\hline & subdiv & 2.5 & 0.492 & 9.4 & 1.256 & 19.3 & 2.728 \\
\hline \multirow[t]{3}{*}{$120 \times 250$} & $2-\mathrm{sec}$ & 1.8 & 0.581 & 4.4 & 1.197 & 37.0 & 8.449 \\
\hline & $3-\sec$ & 1.9 & 0.574 & 3.5 & 0.953 & 112.7 & 19.61 \\
\hline & subdiv & 1.9 & 0.572 & 3.5 & 0.947 & 193.4 & 30.95 \\
\hline \multirow[t]{3}{*}{$150 \times 250$} & $2-\mathrm{sec}$ & 1.2 & 0.763 & 26.4 & 7.554 & 88.8 & 27.75 \\
\hline & $3-\sec$ & 1.2 & 0.770 & 96.0 & 21.63 & 122.4 & 31.76 \\
\hline & subdiv & 1.2 & 0.783 & 176.7 & 37.82 & 262.8 & 64.18 \\
\hline
\end{tabular}

$(m, n, q)=(60,100,30)$. We see from these figures that each code behaves very similarly in response to changes in both $q$ and $\theta$. For each particular $q$ and $\theta$, however, the performance of 2 -sec is slightly superior to the others. This tendency is more pronounced when $q$ increases and $\theta$ decreases. The computational results on larger-scale instances are summarized in Table 1, where the column labeled '\#' shows the average number of iterations and the column labeled 'time' the average CPU time in seconds when $(m, n, q)$ ranged up to $(150,250,125)$, with $\theta$ fixed at 5.0. Since those average values are strongly affected by rare ill-conditioned instances, they do not always respond properly to changes in $(m, n, q)$. For each particular $(m, n, q)$, again, 2-sec performs better than 3-sec and subdiv, especially when the proportion of nonlinear variables $q / n$ is relatively large. 


\section{Concluding remark}

In our previous paper [7], we attempted to prove the convergence of the simplicial algorithm with $\omega$-subdivision along the lines suggested in $[4,17]$. We achieved our aim by relaxing the feasible set $D$ of the target problem (1), but instead allowed an error in the feasibility of the algorithm output within a specified tolerance. In this paper, we have relaxed each simplex $\Delta$ used for subdivision rather than $D$ itself, and consequently managed to ensure the feasibility of the output without much departing from the lines of the convergence proof in [7]. In addition, we have generalized the $\omega$-bisection rule proposed in [7], and established the $\omega$ - $k$-section rule, which subdivides $\Delta$ into at most $k$ subsimplices for a prescribed number $k$. We have shown through numerical comparison that the algorithm incorporating $\omega$ - $k$-section performs as well as the one with the usual $\omega$-subsection when $k=2,3$ and 4 . In particular, the performance of $\omega$-bisection, i.e., $\omega$-k-section for $k=2$, is superior to others, including the usual $\omega$-subdivision. However, we have yet to find a satisfactory explanation for this superiority of $\omega$-bisection, which is a subject for further study.

\section{Acknowledgment}

The authors would like to thank the anonymous referees for their valuable comments, which significantly improved the quality of this article.

\section{References}

[1] GNU Octave, http://www.gnu.org/software/octave/.

[2] Horst, R., "An algorithm for nonconvex programming problems", Mathematical Programming 10 (1976), 312-321.

[3] Horst, R., P.M. Pardalos, and N.V. Thoai, Introduction to Global Optimization, SpringerVerlag (Berlin, 1995).

[4] Horst, R., and H. Tuy, Global Optimization: Deterministic Approaches, 3rd ed., Springer-Verlag (Berlin, 1996).

[5] Jaumard, B., and C. Meyer, "A simplified convergence proof for the cone partitioning algorithm”, Journal of Global Optimization 13 (1998), 407-416.

[6] Jaumard, B., and C. Meyer, "On the convergence of cone splitting algorithms with $\omega$ subdivisions", Journal of Optimization Theory and Applications 110 (2001), 119-144.

[7] Kuno, T., and P.E.K. Buckland, "A convergent simplicial algorithm with $\omega$-subdivision and $\omega$-bisection strategies", Journal of Global Optimization 52 (2012), 371-390.

[8] Kuno, T., and T. Ishihama, "A convergent conical algorithm with $\omega$-bisection for concave minimization", Journal of Global Optimization 61 (2015), 203-220. 
[9] Locatelli, M., "Finiteness of conical algorithm with $\omega$-subdivisions", Mathematical Programming A 85 (1999), 593-616.

[10] Locatelli, M., and U. Raber, "On convergence of the simplicial branch-and-bound algorithm based on $\omega$-subdivisions", Journal of Optimization Theory and Applications 107 (2000), 69-79.

[11] Locatelli, M., and U. Raber, "Finiteness result for the simplicial branch-and-bound algorithm based on $\omega$-subdivisions", Journal of Optimization Theory and Applications 107 (2000), 81-88.

[12] Locatelli, M., and F. Schoen, Global Optimization: Theory, Algorithms, and Applications, SIAM (PA, 2013).

[13] Rockafellar, R.T., Convex Analysis, Princeton University Press (NJ, 1970).

[14] Thoai, N.V., and H. Tuy, "Convergent algorithms for minimizing a concave function", Mathematics of Operations Research 5 (1980), 556-566.

[15] Tuy, H., "Concave programming under linear constraints", Soviet Mathematics 5 (1964), 1437-1440.

[16] Tuy, H., "Normal conical algorithm for concave minimization over polytopes", Mathematical Programming 51 (1991), 229-245.

[17] Tuy, H., Convex Analysis and Global Optimization, Springer-Verlag (Berlin, 1998). 